\title{
Gestaltungs- und Verhandlungsrechte von Beschäftigten
}

Im Windschatten der Infragestellung des Normalarbeitsverhältnisses erhält ein neues arbeitsrechtliches Regelungsmodell immer größere Bedeutung: Durch Gestaltungsansprüche können Beschäftigte vom Arbeitgeber verlangen, dass er das Beschäftigungsverhältnis in bestimmten Teilbereichen entsprechend ihren individuellen Interessen gestaltet. Insbesondere am Beispiel des Anspruchs auf Teilzeitarbeit lässt sich zeigen, welche Chancen und Grenzen solche Gestaltungs- und Verhandlungsansprüche aufweisen und wie sie kollektivrechtlich eingebunden und unterstützt werden könnten.

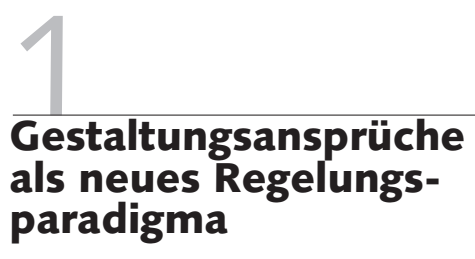

Seit Langem werden traditionelle Strukturen der Arbeitsbeziehungen und des Arbeitsrechts infrage gestellt. Hintergründe sind einerseits eine gesellschaftliche Individualisierung der Lebensverhältnisse und sozialen Identitäten, andererseits eine Flexibilisierung der Produktions- und Beschäftigungsverhältnisse. Das Normalarbeitsverhältnis als Leitbild des Arbeitsrechts kommt so von mehreren Seiten unter Druck.

Parallel sind im Arbeitsrecht Regelungsmodelle entstanden, die vielleicht künftig eine größere Vielfalt an Beschäftigteninteressen in den arbeitsrechtlichen Schutz integrieren könnten. So hat sich im 21. Jahrhundert eine neue Generation von Ansprüchen herausgebildet, mit denen einzelne Beschäftigte vom Arbeitgeber in bestimmten Teilbereichen eine Neugestaltung ihres Beschäftigungsverhältnisses entsprechend ihren individuellen Interessen verlangen können (im Folgenden „Gestaltungsansprüche“ genannt). Zum Beispiel gewähren $₫ 8$ Teilzeit- und Befristungsgesetz (TzBfG) oder $\$ 15$ Abs. 5-7 Bundeselterngeld- und Elternzeitgesetz (BEEG) Ansprüche auf Reduzierung und Neuverteilung der Arbeitszeit. Nach $\$ 81$ Abs. 4 Sozialgesetzbuch (SGB) IX können Schwerbehinderte vom Arbeitgeber fordern, dass die Beschäftigungsbedingungen angepasst werden.

\section{Hintergründe: Kritik an den Leitbildern des Arbeitsrechts}

Die neuen Gestaltungsansprüche sind aus einer Kritik an herkömmlichen Leitbildern des Arbeitsrechts entstanden. Die Aufgabe des Arbeitsrechts besteht ja vor allem darin, die typischen Ungleichgewichte im Beschäftigungsverhältnis zugunsten der Beschäftigteninteressen auszugleichen. Die grundsätzlichen Ungleichgewichte sind unbestreitbar; daraus ergibt sich auch eine Reihe typischer Beschäftigteninteressen. Jenseits eines Kernbereichs der Typizität differenzieren sich die Interessen aber aus. Der Anteil der unbefristet Vollzeitbeschäftigten an der Gesamtzahl der Erwerbstätigen sinkt jedenfalls kontinuierlich (Hoffmann/Walwei 1998). Zwar arbeiten in Deutschland immer noch ca. zwei Drittel der Beschäftigten in einem Normalarbeitsverhältnis. Jedoch fungiert diese Form der Erwerbstätigkeit immer weniger als politisches, und auch immer weniger als individuelles Leitbild: So bemühen sich die europäische und die deutsche Arbeitsmarktpolitik um eine Schaffung von Übergängen zwischen Arbeitsmärkten und Tätigkeiten, um „employability“ über ein bestehendes Arbeitsverhältnis hinaus und um lebenslanges Lernen. Und auch die Menschen gehen in ihren subjektiven Lebensentwürfen ihr Erwerbsleben immer stärker als „Arbeitskraftunternehmer" an (Voß/Pongratz 1998), sehen ihre berufliche Zukunft kaum noch als kontinuierliche Karriere in einem einzigen Unternehmen vor sich und basteln mühsam an der Schaffung einer „Work-Life-Balance“. Die Ressourcen „Verfügung über Zeit“ und „Qualifikation“ ge- winnen neben den Ressourcen „Geld“ und „Gesundheit“ an Bedeutung. Für Menschen mit Fürsorge- und Familienpflichten (also faktisch insbesondere für Frauen) war das Normalarbeitsverhältnis ohnehin nicht geschaffen (Holst/ Maier 1998); weibliche Erwerbsarbeitslebensläufe sind seit jeher „Patch-Work“-Lebensläufe.

\subsection{FLEXICURITY STATT DEREGULIERUNG}

Die Antwort von Deregulierungspolitiken auf die Individualisierung lautet vor allem: „Stärkung“ des individuellen Arbeitsvertrags, Schwächung kollektiver Rechtsquellen und Handlungsinstrumente sowie Durchlöcherung der Normenhierarchie des Arbeitsrechts. Überlässt man die Gestaltung der Erwerbsarbeit auf diese Weise dem Individualarbeitsvertrag und verzichtet damit auf die gesetzliche und kollektivrechtliche Gestaltung der Arbeitsbeziehungen, so verabschiedet man sich letztlich vom Recht als Integrations- und Steuerungsinstrument. Eine formal verstandene Vertragsfreiheit führt in der Erwerbsarbeit nicht zu Verhältnissen, die eine ökonomische oder soziale Gerechtigkeitsvermutung in sich tragen. Daran hat die Individualisierung der Lebens- und Arbeitsverhältnisse nichts geändert - im Gegenteil: Die größere Individualisierung der Lebensverhältnisse bringt nicht weniger, sondern mehr Regelungsbedürfnisse mit sich. Die Schutzbedürfnisse werden nicht weniger,

\footnotetext{
Eva Kocher, Dr. jur., Privatdozentin und Rechtsanwältin in Hamburg; Arbeitsschwerpunkte: Arbeitsrecht, Privatrecht, Verfahrensrecht. e-mail: eva.kocher@wiso.uni-hamburg.de
} 
sondern individueller. Eine immer größere Vielfalt an Beschäftigteninteressen muss sowohl gegenüber Flexibilisierungsinteressen der Arbeitgeber als auch gegenüber dem Normalitätsdruck des Leitbildes „Normalarbeitsverhältnis" geschützt werden.

Der erste Aspekt bedeutet: Die Beschäftigteninteressen an Individualisierung sind nicht notwendig deckungsgleich mit den Flexibilisierungsinteressen der Arbeitgeber. Wo die Produktion immer kundenorientierter und differenzierter, die Zeitplanung immer abhängiger von individuellen Kundenwünschen wird und wo die Anforderungen an lebenslange Qualifizierung und Bildung immer höher werden, besteht die Gefahr, dass individuelle Beschäftigteninteressen an verwertbarer Qualifizierung oder an der Vereinbarkeit von Privat- und Erwerbsleben noch weniger Raum erhalten als im Normalarbeitsverhältnis (Bauer 2001). Die Verteilungskämpfe betreffen so nicht mehr nur das Entgelt, sondern erstrecken sich auf Fragen der Qualifizierung, auf Arbeitsinhalte sowie auf Zeitsouveränität. Flexicurity (Wilthagen/Tros 2004) kann insofern bedeuten, individuelle Beschäftigteninteressen gegen Flexibilisierungsinteressen des Arbeitgebers zu schützen.

\subsection{DISKRIMINIERUNGSVERBOTE UND "ACCOMMODATION"}

Spätestens mit dem Zwang, das Verbot der Diskriminierung wegen des Geschlechts anzuwenden, musste auch im deutschen Arbeitsrecht grundsätzlich hinterfragt werden, inwieweit Arbeitsrecht und andere Normen der Arbeitswelt als Exklusionsmechanismen dienen. Mit dem Allgemeinen Gleichbehandlungsgesetz (AGG) werden die Exklusionswirkungen der Normalitätsannahmen nun auch im deutschen Recht breiter diskutiert und z. B. in Bezug auf ethnische oder sexuelle Minderheiten infrage gestellt. Aus gesellschaftlichen Strukturen der Ungleichheit, Diversität und Diskriminierung ergibt sich eine Vielfalt von Beschäftigteninteressen; diese gilt es gegenüber dem Normalitätsdruck des Leitbildes „Normalarbeitsverhältnis“ zu sichern.

Die Diskriminierungsverbote nehmen zur Kenntnis, dass gleiche Bedingungen und Chancen nur erreicht werden können, wenn bestehende Unterschiede und Herrschaftsverhältnisse berücksichtigt werden. Eine strukturelle Schwäche der Diskriminierungsverbote liegt jedoch darin, dass sie lediglich eine Kritik betrieblicher Normalität ermöglichen, jedoch keine proaktive Veränderung der Arbeitsorganisation oder der Arbeitszeit fördern. Diskriminierungsschutz im engen Sinn zielt auf Gleichbehandlung im vorgegebenen Rahmen. Dies ist für eine wirkliche Integration meist $\mathrm{zu}$ wenig. Beschäftigten mit Familienpflichten wird es wenig helfen, wenn sie die gleichen Chancen in einem Arbeitsverhältnis bekommen, das darauf zugeschnitten ist, dass man dem Unternehmen voll zur Verfügung steht. Behinderten Beschäftigten wird es kaum nützen, die gleichen Chancen in einem Betrieb zu erhalten, der von mindestens durchschnittlicher körperlicher und geistiger Belastbarkeit ausgeht. Ausgehend vom Antidiskriminierungsrecht werden deshalb zunehmend Gleichstellungs- und Gestaltungsansprüche entwickelt (Kocher 2002). Das US-amerikanische und kanadische Antidiskriminierungsrecht kennt mit den Ansprüchen auf „accommodation“, also auf Anpassung, eine eigenständige Kategorie von Gestaltungsansprüchen, die in ihrer Reichweite zwischen dem Diskriminierungsverbot und den positiven Maßnahmen oder Förderpolitiken angesiedelt sind.

Im europäischen und deutschen Recht wird ,accommodation" noch nicht systematisch als eigenständiges Rechtsinstitut im Kontext des Diskriminierungsschutzes diskutiert (siehe aber Waddington/Hendriks 2002). In der Gesetzgebung gibt es jedoch schon erste Ansätze in diese Richtung. Das wichtigste Beispiel ist Art. 5 der gemeinschaftsrechtlichen Richtlinie 2000/78/ EG (Rahmenrichtlinie gegen Diskriminierung am Arbeitsplatz), wonach den Menschen mit Behinderung durch angemessene Vorkehrungen Zugang zur Beschäftigung zu ermöglichen ist. Diese Vorgabe wurde im SGB IX ins deutsche Recht umgesetzt. Schon $\$ 106$ Satz 3 Gewerbeordnung (GewO) verpflichtet den Arbeitgeber, bei der Ausübung des Weisungsrechts auf Behinderungen von Beschäftigten Rücksicht zu nehmen. $\$ 81$ Abs. 4 SGB IX enthält konkrete Anforderungen an Arbeitgeber, bei der Ausgestaltung der Arbeitsorganisation, Arbeitsumgebung und Arbeitsplätze die Belange behinderter Beschäftigter zu beachten.

\section{BEISPIELE}

(1) Schwerbehinderte haben also Ansprüche auf bevorzugte Berücksichtigung bei innerbetrieblichen Bildungsmaßnahmen
(Nr. 2), Erleichterungen zur Teilnahme an außerbetrieblichen Bildungsmaßnahmen (Nr. 3), behinderungsgerechte Anpassung und Ausstattung des Arbeitsplatzes und Arbeitsumfeldes (Nr. 4 und 5) sowie Anspruch auf eine „Beschäftigung, bei der sie ihre Fähigkeiten und Kenntnisse möglichst voll verwerten und weiterentwickeln können" (Nr. 1) (zu Vorgängernormen Welti 2003, S. 447ff). Wenn wegen Art oder Schwere der Behinderung eine kürzere Arbeitszeit notwendig ist, gibt es auch einen Anspruch auf Teilzeitbeschäftigung ( $\$ 81$ Abs. 5 Satz 3 SGB IX ).

(2) $\$ 6$ Abs. 4 Satz 1 Arbeitszeitgesetz (ArbZeitG) ist ein weiteres Beispiel für einen Gestaltungsanspruch, der im Kontext des Diskriminierungsschutzes entstanden ist und als Anrecht auf ,accommodation“ einzuordnen ist. Danach hat der Arbeitgeber einen Nachtarbeitnehmer auf dessen Verlangen auf einen geeigneten Tagesarbeitsplatz umzusetzen. Dieser Anspruch steht nur Beschäftigten zu, die ein Kind unter zwölf Jahren oder einen schwerpflegebedürftigen Angehörigen zu betreuen haben. Die Vorschrift ist an die Stelle des Nachtarbeitsverbots für Frauen getreten, das zum Teil damit gerechtfertigt wurde, dass Frauen häufiger Familienpflichten wahrzunehmen hätten, mit denen ein Nachtarbeitsverbot nicht zu vereinbaren sei. Indem so Frauen wegen ihres Geschlechts von vornherein auf eine bestimmte Geschlechtsrolle festgelegt und von bestimmten Teilen des Arbeitsmarktes ausgeschlossen wurden, handelte es sich um Diskriminierung (BVerfG 1992; EuGH 1991). Auf der anderen Seite führt aber eine Gestaltung von Arbeitsverhältnissen, die auf „private“ Betreuungs-, Fürsorge- und Familienpflichten wiederum gar keine Rücksicht nimmt, ebenfalls (diesmal mittelbar) zu einem Ausschluss von Frauen. Insofern der Umsetzungsanspruch im Arbeitszeitgesetz diese Rücksichtnahme zu gewährleisten versucht, handelt es sich um einen geschlechtsneutralen gleichstellungspolitischen Gestaltungsanspruch.

(3) In einem ähnlichen Kontext steht $\$ 15$ Abs. 5-7 BEEG, wonach während der Elternzeit ein (geschlechtsunabhängiger) Anspruch auf Teilzeitarbeit besteht. Er soll Personen mit Familienpflichten (häufig Frauen) eine Teilhabe an der Erwerbsarbeit ermöglichen, die sich an deren Bedürfnisse nach Zeitsouveränität anpasst. Vorreiter in 
der Begründung von Ansprüchen auf Teilzeitarbeit waren übrigens nicht zufällig die Geschlechtergleichstellungsgesetze im öffentlichen Dienst. $\$ 12$ des BGleiG ${ }^{1}$ lautet z. B.: „Die Dienststelle hat Arbeitszeiten und sonstige Rahmenbedingungen anzubieten, die Frauen und Männern die Vereinbarkeit von Familie und Erwerbstätigkeit erleichtern, soweit zwingende dienstliche Belange nicht entgegenstehen." Auch auf der tariflichen Ebene gibt es besondere Teilzeitansprüche, wie z. B. im öffentlichen Dienst für Beschäftigte, die minderjährige Kinder oder pflegebedürftige Angehörige betreuen $(\$ 15 \mathrm{~b}$ BAT/-O/ $\$ 15 \mathrm{~b}$ MTArb-O bzw. $\$ 11$ TVöD/\$ 11 TV-L).

(4) Das Verbot der Religionsdiskriminierung nach dem AGG ist sicher wichtig. Zur Gewährleistung gleicher Chancen und gleichen Respekts für die Angehörigen aller Religionen im Betrieb reicht ein solches Verbot jedoch nicht aus. Denn häufig ist der betriebliche Arbeitsrhythmus nur an der christlichen Religion ausgerichtet. Vor diesem Hintergrund hat z. B. das Landesarbeitsgericht (LAG) Hamm aus der allgemeinen Rücksichtnahmepflicht des Arbeitgebers und der Religionsfreiheit einen grundsätzlichen Anspruch muslimischer Beschäftigter auf individuelle Gebetspausen hergeleitet: „Der gläubige Arbeitnehmer ist unter Berücksichtigung der betrieblichen Belange ... grundsätzlich berechtigt, seinen Arbeitsplatz zur Abhaltung kurzzeitiger Gebete zu verlassen." (LAG Hamm 2002b). Hier scheint noch eine andere Traditionslinie auf. Denn das LAG begründete sein Urteil letztlich nicht mit Gleichbehandlung, sondern mit der Religionsfreiheit. Mit einer ähnlichen freiheitsrechtlichen Begründung erkennt das Bundesarbeitsgericht (BAG) schon seit Langem individuelle Gewissensentscheidungen im Arbeitsverhältnis an. Es hat zum Beispiel die Arbeitsverweigerung eines Druckers, der kein Werbematerial für „Landser-Literatur" herstellen wollte, für gerechtfertigt gehalten: Im Rahmen seines Weisungsrechts müsse der Arbeitgeber einen ihm offenbarten Gewissenskonflikt des Arbeitnehmers berücksichtigen (BAG 1984).

\subsection{VERALLGEMEINERTE GESTALTUNGSANSPRÜCHE}

Etwas anders gestrickt als diese Gestaltungsansprüche ist der Anspruch auf Reduzierung der Arbeitszeit nach $\$ 8$ TzBfG.
Danach kann jeder Arbeitnehmer und jede Arbeitnehmerin Verringerung (sowie eine bestimmte Verteilung) der Arbeitszeit verlangen. Beschäftigte, die gerne ihre Arbeitszeit reduzieren möchten, müssen hier nicht bestimmte Gründe, wie z. B. Familienpflichten, angeben. Damit löst sich der Anspruch aus dem Begründungszusammenhang des Diskriminierungsschutzes.

$\$ 8$ TzBfG ist unter den Ansprüchen auf Reduzierung der Arbeitszeit der bekannteste. Er trat im Jahre 2001 in Kraft und wurde bereits im selben Jahr von fast 85.000 Beschäftigten geltend gemacht - mit steigender Inanspruchnahme in den Folgejahren (Magvas/Spitznagel 2002; Wanger 2004; Opitz 2005, S. 166). Der Anspruch steht in einem politischen Zusammenhang mit Flexicurity-Politiken. Es ist kein Zufall, dass dieser Teilzeitanspruch in der Struktur einem niederländischen Vorbild folgt (Opitz 2005) - und dass die Niederlande das Heimatland der Flexicurity sind (Wilthagen/ Tros 2004, S. 172 ff.; zum Begründungszusammenhang Diskriminierungsschutz siehe auch Bauer 2001).

Neben der Zeitsouveränität ist die Qualifizierung eine der Fragen, in denen individuelle Interessen von Beschäftigten sich ausdifferenzieren. Es ist insofern nicht überraschend, wenn auch in diesem Bereich Ansätze für die Entwicklung von Gestaltungsansprüchen zu finden sind - allerdings auf niedrigem Niveau. Ergebnis der tarifpolitischen Diskussionen über individuelle Qualifizierungs- und Weiterbildungsansprüche, wie sie Ende der 1990er Jahre stattfanden, waren einige Freistellungsansprüche sowie der Tarifvertrag zur Qualifizierung in der Metall- und Elektroindustrie Baden-Württemberg aus dem Jahre 2001: Er gewährt einen individuellen Anspruch von Beschäftigten auf ein regelmäßiges Gespräch über den Qualifizierungsbedarf - also einen bloßen Verhandlungsanspruch.

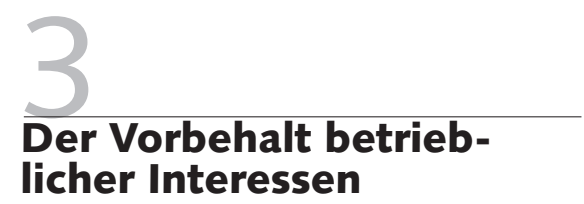

Die Gestaltungsansprüche haben eins gemeinsam: Sie stehen sämtlich unter dem Vorbehalt der Vereinbarkeit mit den betrieblichen Interessen. Was als betriebliche Interessen gilt, die tatsächlich den Gestaltungsanspruch rechtlich zunichte machen können, ist allerdings je nach Regelungsbereich unterschiedlich. Gegen den Anspruch der Schwerbehinderten auf Teilzeitarbeit kann der Arbeitgeber allenfalls „Unzumutbarkeit" einwenden. Ein Arbeitszeitreduzierungsverlangen nach dem Bundeselternzeitgesetz kann vom Arbeitgeber nur innerhalb von vier Wochen aus „dringenden betrieblichen Gründen" schriftlich abgelehnt werden. Der Teilzeitanspruch aus den Tarifverträgen des öffentlichen Dienstes steht unter dem Vorbehalt dienstlicher oder betrieblicher Belange, der aus $\$ 12$ BGleiG unter dem Vorbehalt „zwingender dienstlicher Belange“. Im Arbeitszeitgesetz können dem Verlangen nach Umsetzung auf einen Tagesarbeitsplatz „dringende betriebliche Erfordernisse" entgegengehalten werden. Am niedrigsten sind die Anforderungen beim verallgemeinerten Teilzeitanspruch nach dem Teilzeit- und Befristungsgesetz: Er ist allgemein durch „betriebliche Gründe“ begrenzt. Im Falle des Anspruchs muslimischer Beschäftigter auf regelmäßige Gebetspausen hält das LAG Hamm den Arbeitgeber für ,nicht verpflichtet, Gebetspausen [...] hinzunehmen, wenn hierdurch betriebliche Störungen verursacht werden" (LAG Hamm 2002a). Für den Fall der Gewissensentscheidung eines Beschäftigten hängt die Billigkeit der Weisung des Arbeitgebers von den betrieblichen Erfordernissen ab, insbesondere von den Ausweichmöglichkeiten (BAG 1984).

Schaut man genauer hin, so findet man die Grundstruktur der Gestaltungsansprüche an allen Ecken und Enden des Individualarbeitsrechts wieder, insbesondere im Arbeitsschutz. Auch an den ältesten Anspruch auf Zeitsouveränität im Bundesurlaubsgesetz sei erinnert: „Bei der zeitlichen Festlegung des Urlaubs sind die Urlaubswünsche des Arbeitnehmers zu berücksichtigen, es sei denn, dass ihrer Berücksichtigung dringende betriebliche Belange oder Urlaubswünsche anderer Arbeitnehmer, die unter sozialen Gesichtspunkten den Vorrang verdienen, entgegenstehen “ ( $\$ 7$ Abs. 1 BUrlG ).

\footnotetext{
Bundesgesetz zur Gleichstellung von Frauen und Männern in der Bundesverwaltung und in den Gerichten des Bundes.
} 


\section{1. ÖKONOMISCHE STATT RECHTLICHER RATIONALITÄTEN}

Was dieser Einwand der betrieblichen Gründe konkret bedeutet, ist am Beispiel des Teilzeitanspruches nach $\$ 8$ TzBfG ausführlich diskutiert und teilweise auch schon höchstrichterlich geklärt worden. Nach dem Gesetzeswortlaut liegt ein betrieblicher Grund, der zur Ablehnung des Teilzeitwunsches berechtigt, insbesondere vor, „wenn die Verringerung der Arbeitszeit die Organisation, den Arbeitsablauf oder die Sicherheit im Betrieb wesentlich beeinträchtigt oder unverhältnismäßige Kosten verursacht" ( $\$ 8$ Abs. 4 Satz 2 TzBfG ).

Die Auslegung dieser Vorgaben wirft Probleme auf. Insbesondere fragt sich natürlich, welche betrieblichen Organisationsformen konkret der Teilzeitbeschäftigung entgegengehalten werden können und wann von wesentlicher Beeinträchtigung bzw. unverhältnismäßigen Kosten die Rede sein kann. Eins wird hier aber schon deutlich: Die Struktur der Ansprüche zwingt die Beschäftigten, ihre eigenen individuellen Interessen in ein Verhältnis zu den unternehmerischen und wirtschaftlichen Rahmenbedingungen zu setzen. Die Beschäftigten werden bei der Durchsetzung ihres Anspruchs gezwungen, sich auf die ökonomischen Rationalitäten und Denkweisen einzulassen. ${ }^{2}$ Es geht um ein Beispiel für die Durchlässigkeit von Beschäftigungsverhältnissen gegenüber den Anforderungen des Wettbewerbs.

\subsection{DIE FREIHEIT UNTERNEHME- RISCHER ENTSCHEIDUNG}

Eine wichtige Frage geht dahin, inwieweit die Arbeitsgerichte bei der Auslegung der „betrieblichen Gründe“ organisatorische Konzepte des Arbeitgebers überhaupt auf ihre Zweckmäßigkeit hinterfragen dürfen und inwiefern sie gehalten sind, mögliche Alternativen der Arbeitsorganisation in Betracht zu ziehen (Preis 2006, Rn 23ff.). Das BAG hat bereits in seinen ersten Entscheidungen zu $\$ 8$ TzBfG die gerichtliche Prüfungsbefugnis begrenzt. Die Prüfung, ob betriebliche Gründe vorliegen, erfolgt nun anhand dreier Stufen (BAG 2003a): In der ersten Stufe ist festzustellen, ob überhaupt, und, wenn ja, welches betriebliche Organisationskonzept der vom Arbeitgeber als erforderlich angesehenen Arbeitszeitrege- lung zugrunde liegt. In einer zweiten Stufe ist zu hinterfragen, inwieweit die Arbeitszeitregelung dem Arbeitszeitverlangen des Arbeitnehmers tatsächlich entgegensteht. Wird dies bejaht, ist in einer dritten Stufe das Gewicht der entgegenstehenden betrieblichen Belange abzuwägen: Werden durch die vom Arbeitnehmer gewünschte Arbeitszeit besondere betriebliche Belange oder das betriebliche Organisationskonzept wesentlich beeinträchtigt?

Wesentliche Grenzen der Prüfungsbefugnis liegen auf der ersten Stufe: Die unternehmerische Aufgabenstellung und die daraus abgeleiteten organisatorischen Entscheidungen seien von den Arbeitsgerichten hinzunehmen, soweit sie rational nachvollziehbar seien. Das BAG akzeptierte so z. B. die Vorgabe eines Arbeitgebers in einem heilpädagogischen Kindergarten, die Kinder seien täglich kontinuierlich durch dasselbe Personal zu betreuen, weshalb die Arbeitszeiten den Öffnungszeiten entsprechen müssten (BAG 2003c). Ein anderer Fall: Plane eine Fluggesellschaft in SechsTages-Ketten, so sei dies hinzunehmen, selbst wenn dadurch bestimmte Teilzeitbeschäftigungen unmöglich gemacht würden (BAG 2006a); oder: Lege der Arbeitgeber ein Verkaufsgebiet fest und bestimme gleichzeitig, welche Arbeitszeit hierfür zu veranschlagen ist, dürfe dies zur Folge haben, dass es für Pharmareferenten mit hohem regelmäßigem Fortbildungsbedarf keine Teilzeitbeschäftigung gebe (BAG 2005).

Mit dem Verweis auf „betriebliche Gründe" stoßen die Beschäftigten mit ihren eigenen Interessen also immer wieder an die Grenzen der Freiheit der Unternehmerentscheidung, ${ }^{3}$ ähnliches gilt im Fall der „dringenden betrieblichen Gründe“. Die Verwirklichung der Gestaltungsansprüche ist faktisch und rechtlich davon abhängig, dass der Arbeitgeber bereits ein Organisationskonzept eingeführt hat, das es erlaubt, auf individuelle Beschäftigteninteressen Rücksicht zu nehmen (Kocher 2002, S. 173).

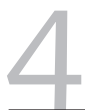 \\ Rechtliche Anforderungen an den Verhandlungs- prozess}

Umso interessanter wird, ob nicht an anderer Stelle eine Hinterfragung der unterneh- merischen Entscheidungen über die Arbeitsorganisation in Betracht kommt. Dies könnte z. B. bei dem neu entwickelten Verhandlungsanspruch im Teilzeit- und Befristungsgesetz der Fall sein.

\subsection{DER VERHANDLUNGSANSPRUCH NACH \& 8 ABS. 3 TZBFG}

Für das innerbetriebliche Verfahren der Auseinandersetzung zwischen Arbeitnehmer und Arbeitgeber über den Teilzeitwunsch sieht $\$ 8$ TzBfG zunächst bestimmte Formen, Fristen und Verfahren vor $(\$ 8$ Abs. 2 und 5 TzBfG ). Darüber hinaus enthält aber Abs. 3 ergänzend einen echten Verhandlungsanspruch. Danach muss der Arbeitgeber mit dem Arbeitnehmer die gewünschte Verringerung der Arbeitszeit erörtern. Das Gesetz schreibt hierfür eine bestimmte Qualität der Verhandlungen und des Austauschs von Argumenten vor: Die Erörterung muss das Ziel verfolgen, „zu einer Vereinbarung zu gelangen“. Die Verhandlungen sollen dem Arbeitgeber durchaus die Chance geben, die Zweckmäßigkeit der unternehmerischen Organisation und ihr Verhältnis zu Teilzeitwünschen der Beschäftigten zu überdenken.

In der Praxis bestehen hier größte Mängel. Noch vor dem Arbeitsgericht scheitern viele Arbeitgeber an der Anforderung, ihre Organisationskonzepte rational nachvollziehbar darzulegen (z. B. BAG 2003a). Sie wollen nicht genau erläutern, welche unternehmerischen Entscheidungen sie getroffen haben, oder können diese auf Nachfrage nicht substanziieren, also sachlich ausführen. Nicht selten verbleibt es bei knappen und pauschalen Hinweisen auf „wirtschaftliche Gründe“; man wolle seine Arbeitsabläufe „bestmöglich“ und „effektiv“ gestalten (BAG 2004; ArbG Hamburg 2006). Die Pflicht zur Durchführung von innerbetrieblichen Verhandlungen wird wohl noch häufiger verletzt; oft werden Anträge der Beschäftigten schlicht schriftlich abgelehnt.

\footnotetext{
2 Siehe z. B. Bröckling/Krasmann/Lemke (2000).

3 Zur rechtlichen Begründung und Notwendigkeit genauer: Hanau 2005, S. 303ff.; genauer zur Reichweite: Bayreuther 2004.
} 


\subsection{RECHTSFOLGEN FEHLENDER ODER FEHLERHAFTER VERHANDLUNGEN}

Verhandlungsrechte und -pflichten werden sich in der betrieblichen Praxis deshalb nur durchsetzen können, wenn sie rechtlich erzwingbar sind und ein Verstoß sanktioniert wird. Die Diskussion darüber, wie eine solche Erzwingbarkeit ausgestaltet werden könnte, steckt in Deutschland erst in den Anfängen. Eine direkte Klage auf „Verhandlungen" ist praktisch wohl kaum sinnvoll. Das BAG hält sie auch rechtlich für ausgeschlossen, denn es ist der Auffassung, es handele sich ohnehin nicht um eine echte Verhandlungspflicht, sondern lediglich um eine beiderseitige Verhandlungsobliegenheit (BAG 2003b).

Man könnte allerdings Entscheidungen, die unter Verletzung von Verfahrensvorschriften zustande gekommen sind, die rechtliche Anerkennung verweigern. So verringert sich die Arbeitszeit automatisch im gewünschten Umfang, wenn sich die Parteien nicht geeinigt haben und der Arbeitgeber nicht wirksam abgelehnt hat (Zustimmungsfiktion) ( $\$ 8$ Abs. 5 Satz 2 TzBfG). Diese Fiktion ließe sich theoretisch auch auf den Fall anwenden, dass die Verfahrenspflichten nicht eingehalten wurden. So war das LAG Düsseldorf vorgegangen (in einem Fall, in dem ein Beschäftigter eine Reduzierung der Arbeitszeit auf Null beantragt hatte, um ein Medizinstudium aufnehmen zu können, LAG Düsseldorf 2002). Das BAG hat jedoch einen anderen Weg eingeschlagen: Das Fehlen einer echten innerbetrieblichen Verhandlung führe nicht dazu, dass die Zustimmung des Arbeitgebers als erteilt gelte (BAG 2003b).

Einzige eigenständige Rechtsfolge für eine Verletzung der Verhandlungsobliegenheit ist es nach Auffassung des BAG, dass der Arbeitgeber im späteren Gerichtsverfahren diejenigen Einwendungen nicht mehr verwenden kann, die im Rahmen einer Verhandlung hätten ausgeräumt werden können (Präklusionswirkung; BAG 2003b). Aber welche Einwendungen könnten das sein? Denn Einwendungen, die ausgeräumt werden können, werden rechtlich ohnehin erfolglos sein. Faktisch kann die Präklusionswirkung immerhin zur Folge haben, dass es dem Arbeitgeber verboten wird, im Gerichtsverfahren betriebliche Gründe nachzuschieben, die er vorher innerbetrieblich nicht erwähnt hatte: Wer in der vorgerichtlichen „Verhandlung“ pau- schal auf „wirtschaftliche Gründe“ verwiesen hat, ohne diese zu spezifizieren, kann dies auch vor Gericht nicht mehr tun (Arbeitsgericht Hamburg 2006 (nicht rechtskräftig); siehe auch Preis 2006, Rn 16).

Das BAG hält die Verhandlungspflicht ohnehin für sekundär. Das Gericht betont, der Arbeitnehmer oder die Arbeitnehmerin könne schließlich unabhängig davon, was vorher im Betrieb geschehen sei, unmittelbar auf die gewünschte Verkürzung der Arbeitszeit klagen (BAG 2003b); dann werden die Argumente eben vor Gericht ausgetauscht.

\section{Kollektivrechtliche Flankierung der Gestaltungsansprüche}

Dahinter steht die Überlegung, dass es den Beschäftigten letztlich doch um die Verkürzung der Arbeitszeit geht. Was haben Beschäftigte davon, dass ein Arbeitgeber ernsthaft mit ihnen verhandelt - wenn sie letztlich doch nicht bekommen, was sie eigentlich wollten? Diese Überlegung übersieht aber zweierlei: Zum einen hat eine betriebliche Kultur, in der Beschäftigte als Subjekte mit ihren individuellen Lebensentwürfen und Wünschen anerkannt werden, einen eigenständigen Wert. Zum anderen aber entfalten Verhandlungen, Partizipation und Kommunikation oft eigene Dynamiken, die über die Effekte von Rechtsansprüchen hinausgehen können. Selbst die rechtlich geschützte unternehmerische Entscheidung kann in solchen Verhandlungen hinterfragt werden.

Insofern ist das Verhandlungsrecht ein Beispiel für eine prozedurale Regelung, das heißt eine Regelung, bei der die Gerichte nicht unmittelbar Rechte zusprechen, sondern nur die Einhaltung von Verfahrensvorgaben und die Qualität der Verhandlungen kontrollieren können (Kocher 2007, S. 83f.). Das Teilzeit- und Befristungsgesetz beschränkt sich zwar nicht auf die prozedurale Regelung, sondern enthält auch ein materielles Recht. Dennoch fragt sich, ob eine prozedurale Regelung in Machtverhältnissen wie dem Beschäftigungsverhältnis überhaupt ein geeignetes zusätzliches Steuerungsinstrument sein kann. Nicht zufällig setzt z. B. die gemeinschaftsrechtliche Richtlinie 2002/14/EG zur Festlegung eines allgemeinen Rahmens für die Unterrich- tung und Anhörung „der Arbeitnehmer“ gerade nicht auf individuelle Information und individuelle Verhandlung, sondern auf die Information und Beratung von Arbeitnehmervertretern. Prozedurale Regelungsinstrumente müssen im Arbeitsrecht auf der Ebene der kollektiven Repräsentation ansetzen.

\subsection{DEFINITION DER BETRIEBLICHEN GRÜNDE}

Die individuellen Gestaltungs- und Verhandlungsansprüche enthalten bereits einige kollektive Elemente. Ein interessantes Modell ist $\$ 83$ SGB IX. Danach trifft der Arbeitgeber mit der Schwerbehindertenvertretung sowie den Betriebsräten (bzw. vergleichbaren betrieblichen Vertretungen) in Zusammenarbeit mit einem speziellen Beauftragten des Arbeitgebers eine Integrationsvereinbarung über die Eingliederung schwerbehinderter Menschen. Die Integrationsvereinbarung enthält Regelungen zur Personalplanung, Arbeitsplatzgestaltung, Arbeitsorganisation, Arbeitszeit, zum Arbeitsumfeld etc.; sie hat bei der Personalplanung besondere Regelungen zur Beschäftigung eines angemessenen Anteils von schwerbehinderten Frauen vorzusehen.

Ein anderes Modell für eine kollektivrechtliche Flankierung findet sich im Teilzeit- und Befristungsgesetz, wonach die Gründe für die Ablehnung von Teilzeitwünschen durch Tarifvertrag festgelegt werden können ( $\$ 8$ Abs. 4 Satz 3 und 4 TzBfG ). Dazu hat der Betriebsrat ohnehin ein Mitbestimmungsrecht über die Lage der Arbeitszeit und damit indirekt über die betrieblichen Gründe. Nach $\$ 6$ ArbZG ist bei der Frage, ob dringende betriebliche Erfordernisse der Umsetzung auf einen Tagesarbeitsplatz entgegenstehen, der Betriebs- bzw. Personalrat zu hören; er kann auch Vorschläge unterbreiten. Dies ist Wirksamkeitsvoraussetzung für die Ablehnung des Versetzungsverlangens aus dringenden betrieblichen Erfordernissen (Wank 2006, Rn 23).

Gemeinsam ist diesen beiden Ansätzen, dass die Mitwirkung der Arbeitnehmervertreter bei der Festlegung der Ablehnungsgründe ansetzt, genauer bei den betrieblichen Gründen. So legen eine Reihe von Tarifverträgen (unter Billigung des BAG) eine Quote von Teilzeitbeschäftigten fest, ab der das Unternehmen als ,"überfordert" anzusehen ist. Damit wird einerseits indirekt eine Mindestquote anerkannt, bis zu deren 
Erreichung Teilzeitarbeit möglich sein muss; andererseits wird es dem Unternehmen ermöglicht, jeden Teilzeitwunsch, der über die Quote hinausgeht, ohne weitere Begründung abzulehnen (BAG 2006b). Problematischer sind Fälle wie der, den das LAG Schleswig-Holstein vor kurzem zu entscheiden hatte: Hier hatte der Betriebsrat seine Zustimmung zum Teilzeitwunsch verweigert und diese Blockadehaltung mit dem „Prinzip“ begründet, Ausnahmen seien grundsätzlich nicht zu machen. Dem hielt das Gericht entgegen, dass es noch kein betrieblicher Grund an sich sei, wenn der Betriebsrat die Zustimmung verweigere (LAG Schleswig-Holstein 2007; Hanau 2005, S. 305). Solche Fälle zeigen, dass die Kollektivparteien ihre Handlungsräume nicht notwendig zugunsten von individuellen Beschäftigteninteressen zu nutzen scheinen, sondern ihre Aufgabe zum Teil noch in der Gewährleistung von Einheitlichkeit sehen. Eine kollektive Absicherung individueller Bedürfnisse ist aber dringend erforderlich (Pfarr 1995).

\subsection{UNTERSTÜTZUNG INDIVIDUELLER VERHANDLUNGEN}

Eine weitere Strategie, individuelle Verhandlungen kollektiv zu flankieren, findet sich im erwähnten Tarifvertrag der Metallindustrie Baden-Württemberg zur Qualifizierung. Er sieht regelmäßige Gespräche zwischen Arbeitgeber und Arbeitnehmer über den Qualifizierungsbedarf vor; bei Nicht-Einigung von Arbeitgeber und Beschäftigten wird eine Kommission eingerichtet, die einen Einigungsversuch unternehmen soll.

Hier wird auf das am weitesten verbreitete Instrument zur Durchsetzung jeder Art von Verhandlungsansprüchen zurückgegriffen: Für den Fall des Scheiterns von Verhandlungen wird eine Schlichtungsoder Schiedsstelle eingesetzt. Im individuellen Verhältnis zwischen Arbeitgeber und Beschäftigten ist dies höchst problematisch; deshalb verbietet das deutsche Arbeitsrecht für den Normalfall die Schlichtung mit Entscheidungsbefugnis (Schiedsgerichtsbarkeit) in arbeitsrechtlichen Individualstreitigkeiten $(\$ \$ 4,101$ Abs. 2 ArbGG). Zulässig bleibt aber eine Schlichtung, wie sie der erwähnte Tarifvertrag vorsieht. Das Beschwerderecht nach $\$ 85$ BetrVG wiederum bietet Möglichkeiten für den Betriebsrat, unternehmerische Entscheidungen im Einzelfall jenseits der rechtlichen Überprüfbarkeit zu hinterfragen - mit kollektiver Absicherung und der Möglichkeit des Schiedsspruchs durch eine
Einigungsstelle. Solche kollektivrechtlichen Unterstützungen von Verhandlungen können prozedural interessante Perspektiven eröffnen.

\section{Fazit}

Gestaltungsansprüche sind rechtliche Instrumente, die von Beschäftigten genutzt werden können, um eine Individualisierung ihres Beschäftigungsverhältnisses im eigenen Interesse und gegen den Willen des Arbeitgebers zu verlangen. Solche Gestaltungsansprüche (wie die Ansprüche von Schwerbehinderten auf Anpassung des Beschäftigungsverhältnisses an die Behinderung oder der Anspruch auf Teilzeitarbeit) stoßen aber rechtlich an enge Grenzen. Die grundsätzlichen Organisationsentscheidungen sind bereits getroffen und werden als unternehmerische Entscheidung von den Arbeitsgerichten rechtlich nicht hinterfragt. Praktisch liegen hier noch Aufgaben für Gewerkschaften und Betriebsräte verborgen; sie können kollektivrechtliche Handlungsspielräume im Interesse individueller Beschäftigter nutzen. 
Bauer, F. (2001): Kann das neue Teilzeit- und Befristungsgesetz die Geschlechterdiskriminierung aufbrechen?, in: WSI-Mitteilungen 8, S. 508513

Bayreuther, F. (2004): Die unternehmerische Entscheidungsfreiheit im Spiegel der aktuellen BAG-Rechtsprechung zum Anspruch auf Teilzeitbeschäftigung, in: Der Betrieb 32, S. 1726-1731

Bröckling, U./Krasmann, S./Lemke, T. (Hrsg.) (2000): Gouvernementalität der Gegenwart. Studien zur Ökonomisierung des Sozialen, Frankfurt am Main

Hanau, H. (2005): Die „, betrieblichen Gründe“ des § 8 Abs. 4 Satz 1 TzBfG im Lichte aktueller Entscheidungen des BAG, in: Recht der Arbeit 5, S. 301-305

Hoffmann, E./Walwei, U. (1998): Normalarbeitsverhältnis: ein Auslaufmodell? Überlegungen zu einem Erklärungsmodell, in: Mitteilungen aus der Arbeitsmarkt- und Berufsforschung 3, S. 409-425

Holst, E/Maier, F. (1998): Normalarbeitsverhältnis und Geschlechterordnung, in: Mitteilungen aus der Arbeitsmarkt- und Berufsforschung 3,

S. 506-518

Kocher, E. (2002): Vom Diskriminierungsverbot zum "Mainstreaming", in: Recht der Arbeit 3, S. 167-173

Kocher, E. (2007): Funktionen der Rechtsprechung, Tübingen

Magvas, E./Spitznagel, E. (2002): Teilzeitarbeit: Neues Gesetz bereits im ersten Jahr einvernehmlich umgesetzt, IAB-Kurzbericht 23, Nürnberg Opitz, H. C. (2005): Der juristische und gesellschaftliche Umgang mit den Ansprüchen auf Teilzeitarbeit in den Niederlanden und in Deutschland, in: Kritische Justiz 2, S. 164-177

Pfarr, H. (1995): Arbeitsrecht: Ein Tanker im Nebel, in: Gewerkschaftliche Monatshefte, S. 633-640

Preis, U. (2006): Kommentierung des $\S 8$ TzBfG, in: Dieterich, T./MüllerGlöge, R./Preis, U./Schaub, G. (Hrsg.): Erfurter Kommentar zum Arbeitsrecht, 6. Aufl., München

Voß, G. G./Pongratz, H. J. (1998): Der Arbeitskraftunternehmer - Eine neue Grundform der Ware Arbeitskraft?, in: Kölner Zeitschrift für Soziologie und Sozialpsychologie 50, S. 131-158

Waddington, L./Hendriks, A. (2002): The expanding Concept of Employment Discrimination in Europe: From Direct and Indirect Discrimination to reasonable Accommodation Discrimination, in: International Journal of Comparative Labour Law and Industrial Relations, S. 403-428 Wanger, S. (2004): Teilzeitarbeit: Ein Gesetz liegt im Trend, IAB-Kurzbericht 18, Nürnberg
Wank, R. (2006): Kommentierung des $\S 6$ ArbZG, in: Dieterich, T./Müller-Glöge, R./Preis, U./Schaub, G. (Hrsg.): Erfurter Kommentar zum Arbeitsrecht, 6. Aufl., München

Welti, F. (2003): Arbeits- und sozialrechtliche Ansprüche behinderter Menschen auf Qualifizierung, in: Arbeit und Recht 12, S. 445-452 Wilthagen, T./Tros, F. (2004): The concept of 'flexicurity': a new approach to regulating employment and labour markets, in: Transfer, S. $166-186$

\section{Rechtsprechung}

Arbeitsgericht Hamburg (2006): Urteil vom 13.10.2006 - 27 Ca 53/06 (nicht rechtskräftig), in: juris-Datenbank

Landesarbeitsgericht (LAG) Düsseldorf (2002): Urteil vom 1.3.2002, in: Der Betrieb 23, S. $1222 \mathrm{ff}$.

Landesarbeitsgericht (LAG) Hamm (2002a): Urteil vom 18.1.2002, in: Neue Juristische Wochenschrift 27, S. 1970

Landesarbeitsgericht (LAG) Hamm (2002b): Urteil vom 26.2.2002, in: Arbeitsrechtliche Praxis 3 zu § 611 BGB Gewissensfreiheit Landesarbeitsgericht (LAG) Schleswig-Holstein (2007): Urteil vom 1.3.2007 - 4 SaGa 1/07, in: juris-Datenbank Bundesarbeitsgericht (BAG) (1984): Urteil vom 20.12.1984, in: BAGE 47, S. 363

Bundesarbeitsgericht (BAG) (2003a): Urteil vom 18.2.2003 - 9 AZR

164/02, in: Arbeitsrechtliche Praxis 2 zu § 8 TzBfG

Bundesarbeitsgericht (BAG) (2003b): Urteil vom 18.2.2003 - 9 AZR 356/02, in: BAGE 105, S. $133 \mathrm{ff}$.

Bundesarbeitsgericht (BAG) (2003c): Urteil vom 19.8.2003, in: Arbeitsrechtliche Praxis 4 zu § 8 TzBfG

Bundesarbeitsgericht (BAG) (2004): Urteil vom 20.7.2004, in: Arbeitsrechtliche Praxis $11 \mathrm{zu} \S 8 \mathrm{TzBfG}$

Bundesarbeitsgericht (BAG) (2005): Urteil vom 21.6.2005, in: Arbeitsrechtliche Praxis 14 zu § 8 TzBfG

Bundesarbeitsgericht (BAG) (2006a): Urteil vom 15.8.2006, in: Arbeitsrechtliche Praxis 16 zu § 8 TzBfG

Bundesarbeitsgericht (BAG) (2006b): Urteil vom 21.11.2006, in: Betriebs-Berater 18, 2007, S. $1001 \mathrm{ff}$.

Bundesverfassungsgericht (BVerfG) (1992): Urteil vom 28.1.1992, in: BVerfGE 85, $191 \mathrm{ff}$.

Europäischer Gerichtshof (EuGH) (1991): Urteil vom 25.7.1991, in: Arbeitsrechtliche Praxis 28 zu Art 119 EWG-Vertrag 